\section{Authors' reply}

David Torgerson raises the reasonable concern that the single randomised consent design is prone to a dilution effect because of subjects refusing treatment, which should be factored into initial power calculations. He suggests that, had we done so, we would have needed to increase our sample size by $30 \%$.

Two issues are salient-sample size and refusal rate. Our choice of 240 as a target sample size was $20 \%$ more than required to show significant differences in length of stay-not quite the 30\% Torgerson recommends but not far from it. As described in our paper, we made this allowance for several reasons. We acknowledge the potential dilution effect associated with Zelen's design, but believe that our 20\% margin of safety was sufficient to yield meaningful estimates of differences in length of stay.

Moreover, the adjustment recommended by Torgerson is based on an assumed refusal rate of $10 \%$. The relevance of cancer patients' refusal rates to that antici- pated for post-acute general medical patients is not immediately obvious, although the numbers are interesting. In our study the actual refusal rate was $5 \%$ (6/119), a figure described by Parmar as "a good approximation in most [conventional trial] situations." Parmar further notes that if refusal rates are low, approaching those occurring after randomisation in conventional trials, there may be no practical difference between randomised consent and a standard design with regard to dilution.

In all intention to treat analyses, any non-significant finding can constitute a type II error. However, in this study we see no additional risk accruing as a result of the randomised consent design.

1. Parmar MKB. Randomization before consent: practical and ethical considerations. In: Williams CJ, ed. Introducing new treatments for cancer: practical, ethical and legal problems. Chichester: John Wiley and Sons, 1992:194. $d x$

Boston

Collaborative Drug Surveillance

Program, Boston

University School of

Medicine,

11 Muzzey Street,

Lexington, MA

02421, USA

James A Kaye

epidemiologist

Maria del Mar

Melero-Montes

epidemiologist

Hershel Jick

associate professor of

medicine

Correspondence to:

J A Kaye

jkaye@narsil.com

BMJ 2001;322:460-3

\author{
Abstract \\ Objective To estimate changes in the risk of autism \\ and assess the relation of autism to the mumps, \\ measles, and rubella (MMR) vaccine. \\ Design Time trend analysis of data from the UK \\ general practice research database (GPRD). \\ Setting General practices in the United Kingdom. \\ Subjects Children aged 12 years or younger \\ diagnosed with autism 1988-99, with further analysis \\ of boys aged 2 to 5 years born 1988-93. \\ Main outcome measures Annual and age specific \\ incidence for first recorded diagnoses of autism (that \\ is, when the diagnosis of autism was first recorded) in \\ the children aged 12 years or younger; annual, birth \\ cohort specific risk of autism diagnosed in the 2 to 5 \\ year old boys; coverage (prevalence) of MMR \\ vaccination in the same birth cohorts. \\ Results The incidence of newly diagnosed autism \\ increased sevenfold, from 0.3 per 10000 person years \\ in 1988 to 2.1 per 10000 person years in 1999. The \\ peak incidence was among 3 and 4 year olds, and 83\% \\ (254/305) of cases were boys. In an annual birth \\ cohort analysis of 114 boys born in 1988-93, the risk \\ of autism in 2 to 5 year old boys increased nearly \\ fourfold over time, from 8 (95\% confidence interval 4 \\ to 14) per 10000 for boys born in 1988 to 29 (20 to \\ 43) per 10000 for boys born in 1993. For the same \\ annual birth cohorts the prevalence of MMR \\ vaccination was over $95 \%$.
}

Conclusions Because the incidence of autism among 2 to 5 year olds increased markedly among boys born in each year separately from 1988 to 1993 while MMR vaccine coverage was over $95 \%$ for successive annual birth cohorts, the data provide evidence that no correlation exists between the prevalence of MMR vaccination and the rapid increase in the risk of autism over time. The explanation for the marked increase in risk of the diagnosis of autism in the past decade remains uncertain.

\section{Introduction}

The possibility that the mumps, measles, and rubella (MMR) vaccine may be causally related to the risk of autism is currently causing substantial concern. This proposition originated primarily from a publication by Wakefield et al in 1998 that described 12 case reports of children who were diagnosed with ileal-lymphoidnodular hyperplasia followed by behaviour disorders that were clinically diagnosed as representing autism. ${ }^{1}$ In eight of 12 children the behaviour disorder was "linked" in time with MMR vaccination by the parents or the child's physician.

In June 1999 Taylor et al published in the Lancet the results of a study in which they identified children diagnosed as having autism in the North East Thames region for birth cohorts from 1979 to 1992.. They reported that the incidence of autism started to increase in children born in the late 1980s and 
increased dramatically in those born from 1989 to 1992. They also provided estimates of the coverage (prevalence) of MMR vaccination from 1987 to 1995, which rose to over $90 \%$ by $1988-9$. They found no temporal association between MMR vaccination and the incidence of autism within one to two years of vaccination, and there was no "clustering" of cases in the two to four months after vaccination.

In a subsequent letter to the Lancet's editor Wakefield described the study by Taylor et $\mathrm{al}^{2}$ as containing a "fundamental flaw" and cited data from the United Kingdom (north west London) and the United States (California) based on the time trend of autism occurrence by birth cohort in relation to the introduction of the MMR vaccine. ${ }^{3}$ In both areas a dramatic increase in the incidence of autism was reported in temporal association with the rapid introduction of the vaccine.

We used the UK general practice research database (GPRD) to evaluate further the temporal relation of MMR vaccine and the incidence of autism.

\section{Subjects and methods}

The data in the UK general practice research database are firmly established in numerous publications to be of high quality and completeness ${ }^{4}$ and, in particular, the recording of vaccinations in this database has been found to be virtually complete ( $\mathrm{H}$ Jick et al unpublished data). ${ }^{5}$ We initially tried to conduct a casecontrol analysis comparing children who received the MMR vaccine and those not vaccinated in relation to the diagnosis of autism. Only about 3\% of cases and controls, however, did not receive the vaccine, and therefore there was too little information to provide a meaningful estimate of relative odds. We therefore conducted a time trend analysis to explore the relation of the MMR vaccine and the diagnosis of autism over time.

We identified 305 children aged 12 or younger whose diagnosis of autism was first recorded (first recorded diagnosis) during the years 1988 to 1999 (from among 3092742 person years of observation in the base population). We reviewed the detailed computer recorded information for each of these children. We estimated annual incidence (regardless of age at first recorded diagnosis) and age specific incidence (regardless of year of first recorded diagnosis). Some practices stopped providing information before 1999, and therefore the person time available in the later years was smaller than that in the earlier years.

Subsequent analyses were restricted to 114 boys born in 1988-93 who had a first recorded diagnosis of autism at age 2 to 5 years (24-71 months) - that is, during 1990-9. Annual birth cohorts were analysed separately. For each annual birth cohort, we estimated the four year cumulative incidence (risk) of diagnosed autism with the exponential formula: cumulative incidence $=1-\exp \left(-\Sigma I_{k} \Delta t\right)$, where $I_{k}$ represents the estimated age specific annual incidences for the individual birth cohort and $\Delta \mathrm{t}$ is one year. The prevalence of MMR vaccination among children registered in the general practice research database within 60 days of birth who had at least two years of recorded follow up was also calculated separately for each annual birth cohort. Statistical analyses were per-

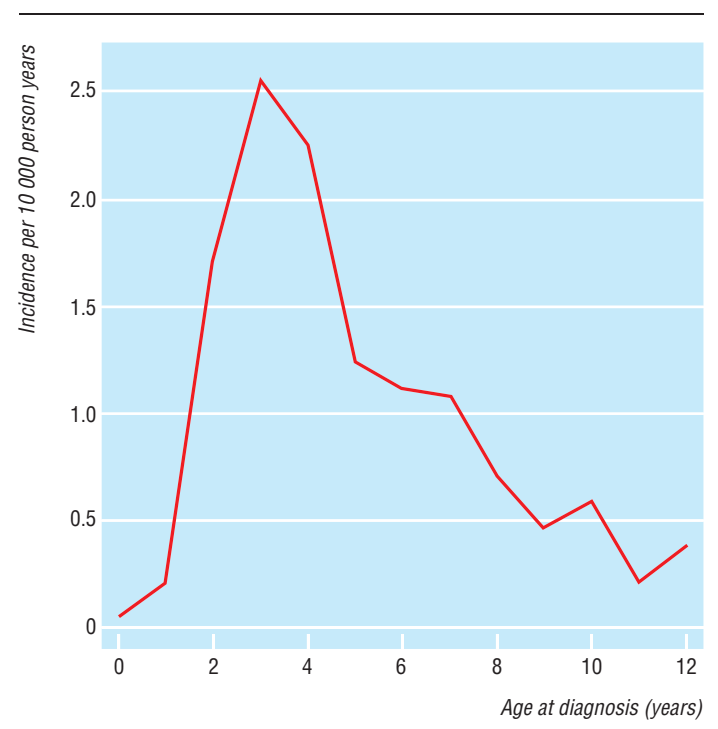

Fig 1 Incidence of autism in children registered in UK general practice research database, by age at diagnosis (total observation time was 3092744 person years)

formed using STATA, version 7.0 (Stata Corporation, College Station, Texas).

\section{Results}

The estimated yearly incidence of diagnosed autism among children aged 12 years or younger (305 cases) increased sevenfold, from 0.3 per 10000 person years in 1988 to 2.1 per 10000 person years in 1999. The median age at first recorded diagnosis of autism was 4.6 years and did not vary substantially over time (table). The peak ages at first recorded diagnosis were 3 years and 4 years (fig 1). Two hundred and fifty four of the cases were male. About 81\% (248/305) of the cases were referred to a specialist for evaluation of the diagnosis.

To assess more precisely the possibility of a temporal association between MMR vaccination and the risk of autism, we analysed data for consecutive annual birth cohorts of boys born during 1988-93. For each annual birth cohort, we estimated the four year cumulative incidence (risk) of a first recorded diagnosis of autism at age 2-5 years. One hundred and fourteen

Number of cases, person years at risk, and mean age of cases, according to year of diagnosis of autism in 305 children aged 12 years or younger.

\begin{tabular}{lcccc}
$\begin{array}{l}\text { Year of } \\
\text { diagnosis }\end{array}$ & $\begin{array}{c}\text { No of } \\
\text { cases }\end{array}$ & $\begin{array}{c}\text { No of person } \\
\text { years at risk }\end{array}$ & $\begin{array}{c}\text { Estimated incidence } \\
\text { per } \mathbf{1 0} \text { 000 person } \\
\text { years }\end{array}$ & $\begin{array}{c}\text { Median age } \\
\text { of cases } \\
\text { (years) }\end{array}$ \\
\hline 1988 & 7 & 255771 & 0.3 & 6.0 \\
\hline 1989 & 8 & 276644 & 0.3 & 5.6 \\
\hline 1990 & 16 & 295901 & 0.5 & 5.0 \\
\hline 1991 & 14 & 309682 & 0.5 & 4.4 \\
\hline 1992 & 20 & 316457 & 0.6 & 4.0 \\
\hline 1993 & 35 & 316802 & 1.1 & 5.8 \\
\hline 1994 & 29 & 318305 & 0.9 & 4.6 \\
\hline 1995 & 46 & 303544 & 1.5 & 4.3 \\
\hline 1996 & 36 & 260644 & 1.4 & 4.7 \\
\hline 1997 & 47 & 216826 & 2.2 & 4.3 \\
\hline 1998 & 34 & 161664 & 2.1 & 5.4 \\
\hline 1999 & 13 & 60502 & 2.1 & 5.9 \\
\hline Total & 305 & 3092742 & 1.0 & 4.6 \\
\hline
\end{tabular}


boys were included in this analysis. The four year risk of diagnosed autism increased nearly fourfold, from 8 (95\% confidence interval 4 to 14) per 10000 for boys born in 1988 to 29 (20 to 43) per 10000 for boys born in $1993(\mathrm{P}<0.0001$ by score test for trend in odds (fig 2)). In contrast, the prevalence of MMR vaccination among children registered in the general practice research database with at least two years of follow up was virtually constant (about 97\%) for each successive annual birth cohort and was similar among males and females (data not shown).

Among the vaccinated children, the median age at first MMR vaccination was 14 months, and $95 \%$ of those vaccinated received their first MMR vaccination by age 20 months. Among 110 cases of autism in boys aged 2 to 5 years born in 1988-93 for whom MMR vaccination could be assessed, the distribution of age at first MMR vaccination was nearly identical to that of the population as a whole, and 109 (99\%) were vaccinated, a prevalence nearly identical to that in the general population.

\section{Discussion}

Previous publications have reported that the overall incidence of clinically diagnosed autism began to rise in the late 1980s, and that the incidence occurs predominantly in boys. ${ }^{236}$ This study shows that the incidence has continued to increase during the past decade. Our analysis of the risk of diagnosed autism for boys aged 2 to 5 years showed a progressive increase for each successive birth cohort from 1988 to 1993 , during which time the prevalence of MMR vaccination was over $95 \%$. It should be noted that the MMR vaccine is given first at about 15 months of age and that autism is not typically diagnosed until age 2 years or later.

If the MMR vaccine were a major cause of the increasing incidence of autism then the risk of autism in successive birth cohorts would be expected to stop rising within a few years of the vaccine being in full use. This was not the case in our study as the cumulative incidence for boys ages 2 to 5 years rose almost fourfold in the 1993 birth cohort (with follow up to 1999) compared with the 1988 birth cohort, whereas the prevalence of MMR vaccination was over $95 \%$.

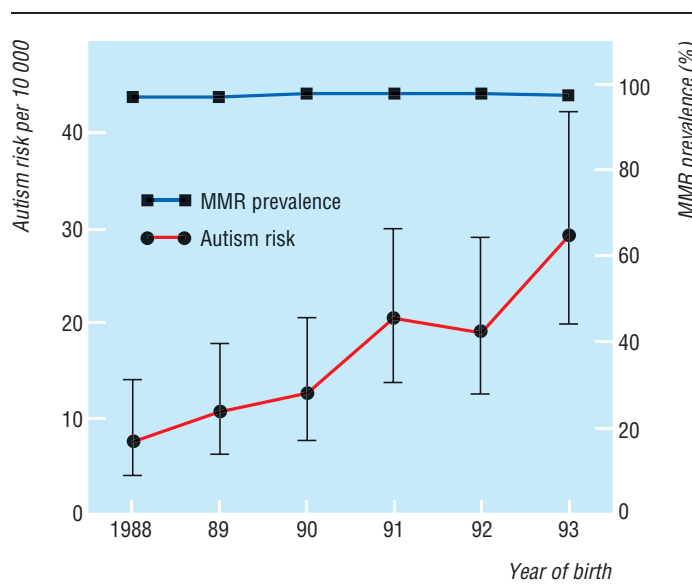

Fig 2 Four year risk of autism (with 95\% confidence intervals) among boys aged 2 to 5 years and prevalence of MMR vaccine, by annual birth cohort

\section{What is already known on this topic}

The incidence of autism in the United Kingdom has increased markedly over the past decade

Some have proposed that this may be related to introduction of the mumps, measles, and rubella (MMR) vaccine in 1988

\section{What this study adds}

The risk of autism increased nearly fourfold among boys aged 2 to 5 years born in 1988-93 and registered in the UK general practice research database, whereas the prevalence of MMR vaccination was over $95 \%$ and virtually constant

These data provide evidence against a causal association between MMR vaccination and the risk of autism

Thus no time correlation exists between the prevalence of MMR vaccination and the incidence of autism in each birth cohort from 1988 to 1993.

We recognise that the diagnosis of autism in our study was not confirmed from original records but consider that differential misclassification of the diagnosis in vaccinated and unvaccinated children is unlikely to vary over the period of the study.

Time trend analysis for the evaluation of the relation of an exposure to an illness is a relatively crude method. This is particularly true where the exposure and the illness are both rising during the period of study as such a correlation may be coincidental and due to changes in other factors that are correlated over time with the outcome illness. Nevertheless, when the incidence of an illness is rising rapidly in each birth year cohort at the same time that an exposure is steady and almost universal, the exposure cannot be the explanation for the rapid increase in incidence that was observed.

The increase in recorded diagnoses of autism that we observed in the UK general practice research database could be due to increased awareness of the condition among parents and general practitioners, changing diagnostic criteria, or environmental factors not yet identified. A strength of our study is that we were able to use population based data in the general practice research database to estimate the birth cohort specific incidence of autism recorded by general practitioners as well as the prevalence of MMR vaccination. A limitation is that we have not yet obtained and evaluated full clinical record information from general practitioners to describe more fully the characteristics of children diagnosed as having autism and to explore other possible explanations for the marked increase in the incidence of this illness during the past decade. Nevertheless, these results provide evidence against a causal relation between MMR vaccination and the risk of autism.

We appreciate the helpful comments of Alexander M Walker on an earlier draft of the manuscript and thank the general practitioners who contribute data to the general practice research database for their excellent ongoing participation and patient care.

Contributors: JAK participated in the study design, analysed the data, and drafted the manuscript. MdelMM-M helped in 
assembling the data for analysis and contributed to writing the manuscript. HJ participated in the study design and revising the manuscript. JAK and $\mathrm{HJ}$ are guarantors for the paper.

Funding: No specific funding.

Competing interests: The Boston Collaborative Drug Surveillance Program is supported in part by grants from AstraZeneca, Berlex Laboratories, Boehringer Ingelheim Pharmaceuticals, Boots Healthcare International, Bristol-Myers Squibb Pharmaceutical Research Institute, GlaxoWellcome, Hoffmann-La Roche, Janssen Pharmaceutica Products, R W Johnson Pharmaceutical Research Institute; McNeil Consumer Products, and Novartis Farmaceutica. JAK is a John and Virginia Taplin fellow at the Harvard School of Public Health and is supported by a training fellowship in cancer epidemiology from the National Cancer Institute (T32-CA 09001).

1 Wakefield AJ, Murch SH, Anthony A, Linell J, Casson DM, Malik M, et al. Ileal-lymphoid-nodular hyperplasia, non-specific colitis, and pervasive developmental disorder in children. Lancet 1998;351:637-41.

2 Taylor B, Miller E, Farrington CP, Petropoulos M-C, Favot-Mayaud I, Li J, et al. Autism and measles, mumps, and rubella vaccine: no epidemiological evidence for a causal association. Lancet 1999;353:2026-9.

Wakefield AJ. MMR vaccination and autism. Lancet 1999:354:949-50.

4 Jick H. A database worth saving. Lancet 1997;350:1045-6.

4 Jick H. A database worth saving. Lancet 1997;350:1045-6. Jick H, Withers JM, Dean
Gen Pract 1995;45:107.

6 Wing L. The autistic spectrum. Lancet 1997;350:1761-6.

(Accepted 8 February 2000)

\title{
What parents are told after prenatal diagnosis of a sex chromosome abnormality: interview and questionnaire study
}

\author{
Lenore Abramsky, Sue Hall, Judith Levitan, Theresa M Marteau
}

\begin{abstract}
Objective To investigate how the prenatal diagnosis of a sex chromosome anomaly is first communicated to parents.

Design Health professionals were interviewed by telephone and the conversation was taped; parents were sent questionnaires at 1 month after diagnosis and those who responded were sent another at 6 months.

Participants 29 health professionals who had recently informed parents that a sex chromosome anomaly had been identified in an apparently anatomically normal, viable fetus. 23 mothers and partners who had been informed of such a diagnosis.

Main outcome measures Health professionals' knowledge about sex chromosome anomalies and parents' responses to information provided by health professionals.

Results Analysis of the telephone interviews identified great variation in what different healthcare professionals know, think, and say about the same sex chromosome anomaly. The small numbers and the low response rate for the questionnaire (39\% for women and 30\% for men) meant that statistical analysis was not appropriate.

Conclusions It is essential for obstetric units to have an established protocol for giving results and for all staff who communicate results to parents to have accurate, up to date information about the condition identified.
\end{abstract}

\section{Introduction}

Most fetuses and babies with an extra sex chromosome are not identified ${ }^{1}$ because there are usually no indications for karyotyping. ${ }^{2-6}$ However, a sex chromosome anomaly is sometimes detected prenatally when amniocentesis is performed to exclude Down's syndrome or other serious chromosomal anomalies. Conditions in which there is an extra sex chromosome are fundamentally different from those such as Down's syndrome in which affected individuals have recognisable characteristics that can be explained by laboratory findings. Prenatal detection of sex chromosome anomalies and other karyotypes or genotypes that may have no phenotypic consequences or only mild ones will become more common as testing becomes more widely available. ${ }^{7}$ Understanding the difficulties faced by health professionals in disclosing the prenatal diagnosis of a sex chromosome anomaly can highlight some of the problems that may be encountered during the prenatal detection of other atypical laboratory findings in fetuses with phenotypes that are likely to fall within the normal range (table 1).

We investigated how the prenatal diagnosis of a sex chromosome anomaly is first communicated to parents. We did not test a hypothesis but hoped to generate discussion about this issue. The first communication to parents is important because it may affect how information presented later is interpreted or even whether it is sought. ${ }^{8-10}$ Previous studies have looked at counselling that occurred once clinical geneticists
Editorial

by Biesecker

North Thames Perinatal Public Health Unit, Department of Medical and Community Genetics, Imperial College of Science, Technology, and Medicine,

Northwick Park

Site, Harrow

HA1 3UJ

Lenore Abramsky senior research officer

Judith Levitan research assistant

Psychology and Genetics Research Group, Guy's, King's College, and St Thomas's Hospitals School of Medicine, London SE1 9RT

Sue Hal research associate Theresa M Marteau professor

Correspondence to: L Abramsky

l.abramsky@ic.ac.uk

BMJ 2001;322:463-6

Table 1 Fetal karyotypes and characteristics ${ }^{2-6}$

\begin{tabular}{|c|c|c|c|c|c|}
\hline \multirow[b]{2}{*}{ Condition } & \multirow[b]{2}{*}{ Frequency } & \multicolumn{3}{|c|}{ Characteristics } & \multirow[b]{2}{*}{ Treatment } \\
\hline & & Intelligence quotient & Fertility & Other considerations & \\
\hline 47XXY (Klinefelter's syndrome) & 1 in 800 males & About $10-15$ points lower than siblings & Infertile & May have gynaecomastia & $\begin{array}{l}\text { Testosterone from } \\
\text { puberty }\end{array}$ \\
\hline 47XXX (Triple X) & 1 in 1000 females & About 20 points lower than siblings & Fertile & None & None \\
\hline 45X (Turner's syndrome)* & 1 in 2500 females & Unaffected & Infertile & $\begin{array}{l}\text { Short stature; may have } \\
\text { congenital malformations }\end{array}$ & $\begin{array}{l}\text { Oestrogen from puberty; } \\
\text { growth hormone }\end{array}$ \\
\hline
\end{tabular}

${ }^{\star}$ Turner's syndrome is common at conception but $98 \%$ of fetuses will be miscarried. 